\title{
Actitud hacia las Matemáticas en Estudiantes de Secundaria: El caso de una Escuela Bilingüe
}

\section{Información de artículo:}

Recibido: 30/03/2021

Aprobado: 30/05/2021

\section{Palabras claves:}

Actitudes, Interés, Utilidad, Autoconcepto, Educación Bilingüe.

\section{Keywords:}

Attitudes, Interest, Utility, Selfconcept, Bilingual Education.

\section{Resumen}

Esta investigación ha determinado las actitudes hacia las matemáticas de los estudiantes de Educación Secundaria de Dowal School en Tegucigalpa-Honduras. Se trata de una investigación cuantitativa sustentada en un diseño "Ex Post Facto" donde se suministró una escala de actitudes a 163 estudiantes de séptimo a undécimo grado en un contexto bilingüe. Los resultados comunican que los estudiantes tienen un autoconcepto y utilidad positiva hacia las matemáticas, aunque, un poco interés hacia el aprendizaje de esta materia. Se concluye que los estudiantes presentan características homogéneas y que de forma global presentan una actitud positiva hacia las matemáticas.

\section{Attitude towards math in Middle and High School students: The case of a bilingual school}

\section{Abstract}

This research has determined the attitudes towards mathematics of Secondary Education students from Dowal School in Tegucigalpa-Honduras. This is a quantitative research based on an "Ex Post Facto" design where an attitude scale was provided to 163 students from seventh to eleventh grade in a bilingual context. The results communicate that the students have a positive self-concept and utility towards mathematics, although, a little interest towards learning this subject. It is concluded that the students present homogeneous characteristics and that in a global way they present a positive attitude towards mathematics.

\footnotetext{
${ }^{1}$ Licenciado en Matemáticas. Profesora de Matemáticas Dowal School, i.rodriguez2511@gmail.com - (D): https://orcid. org/0000-0003-2093-8867

2 Licenciado en Matemáticas. Profesor de Matemáticas Dowal School, levindasayev@gmail.com - (D) $\underline{\text { https://orcid. }}$ org/0000-0003-3904-1304
} 


\section{Introducción}

Es de dominio público que las actitudes median la forma en la que se perciben las circunstancias y en todos los ámbitos de la vida. Desde una perspectiva científica se ha comprobado que las actitudes hacia las matemáticas se consideran parte de la dimensión afectiva de las procesos de estudio matemático, la cual incluye la motivación, la emoción y la ansiedad matemática (Evans, 2006 citado en Yáñez-Marquina \& Villardón-Gallego, 2016). De acuerdo con Jiménez-Bonilla \& Flores-López (2019) "el estudio de las actitudes hacia las matemáticas es fundamental porque impiden una progresión en el aprendizaje de las matemáticas", ya que estas se relacionan con los factores emotivos, académicos, motivacionales, normativos y profesionales (FloresLópez et al., 2018; García-Soto et al., 2018).

En ciertas áreas del conocimiento, como las matemáticas, los estudiantes muestran rechazo al trabajo o participación en las actividades, argumentan bloqueos o se resisten incluso a prestar atención. Según Solís (2017) un buen docente debe tener en cuenta que en lo cognitivo existen también elementos relacionados con las emociones, sensaciones y valores que deben ser inspirados por él, inicialmente siendo empático con las necesidades de sus estudiantes y no se puede ser empático desconociendo las mismas. En este contexto, Rocha-Feregrino, Fuchs-Gómez, \& Juárez-López (2021) se debe reflexionar constantemente sobre las razones del fracaso académico y las motivaciones o razones por las que los estudiantes las consideran difíciles de entender pues se debe encontrar un modelo de aprendizaje que permita verdadera cercanía entre las actitudes y su relación con el aprendizaje de las matemáticas.

El interés de los autores de este artículo, debido a la trayectoria individual en centros educativos de educación privada, es comprender mejor a los estudiantes para ayudarles a mejorar su proceso de aprendizaje ya que a pesar de contar con algunos recursos de aprendizaje que no están presentes en el sistema público no se escapa de tener una alta tasa de reprobación en ocasiones casos de estudiantes con muestras conductuales de completa apatía por la clase, por consiguiente, el estudio de las actitudes hacia el aprendizaje de las matemáticas juega un papel fundamental pues constituye un criterio de autoevaluación sobre la efectividad de las metodologías aplicadas al proceso de enseñanza en la institución.

\section{Literatura}

En el ejercicio de la docencia es muy frecuente el interés por comprender cómo los factores relacionados con la dimensión afectiva ejercen una influencia, directa muchas veces, sobre el desempeño académico de los estudiantes. Es bastante común encontrarse con docentes conversando sobre la actitud, sea esta positiva o negativa de los chicos cuando llega el momento de la clase de matemáticas. Según Ignacio et al. (2006) los estudios de la dimensión afectiva fueron por un largo período de tiempo 
limitados a la investigación de las actitudes, sin embargo, existió un problema persistente para definirlas de forma concisa. Precisamente basados en su utilidad para la vida en la sociedad cada vez más compleja que nos rodea y la vibrante avanzada del uso de tecnología en la actividad educativa se pensaría que las actitudes de los estudiantes deberían ser positivas, pero algunos de los docentes han escuchado alguna vez frases como "es muy difícil", "es aburrido", "No sé cómo puede gustarle", "Ni sé cuándo voy a usar esto".

Entonces, la actitud se entenderá como una predisposición evaluativa (positiva o negativa) que determina las intenciones personales e influye en el comportamiento (Caballero-Carrasco, Blanco-Nieto \& Guerrero-Barona, 2008). Intenciones que incluyen las creencias, convicciones y sentimientos que provocan reacción a un estímulo, en nuestro caso hacia el aprendizaje de las matemáticas. Dichas actitudes se evalúan con base en tres componentes autoconcepto, interés por las matemáticas, y utilidad percibida de las matemáticas (Yáñez-Marquina \& Villardón-Gallego, 2016).

También deben considerarse los resultados previos sobre el desempeño académico que presentan los estudiantes. En Honduras el 21\% de los estudiantes solamente alcanzan niveles bajos de desempeño, $4 \%$ se encuentra en un nivel intermedio y solamente el 1\% en el alto (TIMSS \& PIRLS International Study Center, 2011). Cabe notar que el porcentaje restante se encuentran como datos inconsistentes que no admiten una evaluación apropiada. Resultados posteriores en la aplicación de pruebas PISA del año 2017 muestra que solamente el 15\% de los jóvenes alcanzan el nivel mínimo de competencias en esta escala, sin embargo, un $89 \%$ del total de los participantes tienen una percepción positiva de sus docentes (PISA, 2018, p. 15).

En el modelo del Programa para la Evaluación Internacional de Estudiantes para el Desarrollo (PISA-D por sus siglas en inglés Programme for International Student Assessment-for Development) se consideran las actitudes hacía la escuela y el aprendizaje como uno de los fundamentos del Modelo de Prosperidad Educativa propuesto por la (OCDE, 2018), sin embargo, las puntuaciones de Honduras son inferiores a la media de los países miembros Organización para la Cooperación y Desarrollo Económico (OCDE, 2018), estando 147 puntos por debajo del promedio regional lo que equivale a más o menos 4 años de escolaridad previo a la pandemia. Considerando que tanto el sistema de educación público como privado participan de la aplicación de estas pruebas parece meritorio hacer un estudio a pequeña escala, primero que nada, porque sus resultados pueden convertirse en una herramienta para construir estrategias, repensar las metodologías actualmente aplicadas en un intento por mejorar el rendimiento académico de los estudiantes en la clase de matemáticas.

Para lograr esta mejora en el desempeño se debe contribuir al desarrollo de actitudes positivas hacia el aprendizaje de las matemáticas para que el estudiante logre sentirse capaz de hacer uso del conocimiento de estas si así lo necesita (Rocha-Feregrino, 
Fuchs-Gómez \& Juárez-López, 2021). Una predisposición positiva permite a los estudiantes incursionar en las actividades de tipo colaborativo con mayor fluidez, especialmente tomando en cuenta que con la modalidad de aprendizaje remoto que actualmente está en ejecución en las instituciones debido a la pandemia, se hace uso de múltiples recursos tecnológicos y medios digitales a su disposición para facilitar la comprensión.

\section{Metodología de investigación}

\subsection{Finalidad y objetivos del estudio}

Esta investigación tiene por objetivo general determinar las actitudes hacia las matemáticas de los estudiantes secundaria de Dowal School en Tegucigalpa, tomando en consideración las variable género, edad y grado académico de los estudiantes, por tales razones, se proponen los objetivos específicos siguientes: (1) Identificar las actitudes hacia las matemáticas del estudiantado; (2) Explicar la relación entre los factores actitudinales hacia las matemáticas del estudiantado; (3) Comprobar si existen diferencias significativas entre género, edad, grado y sección en relación con las actitudes hacia las matemáticas del estudiantado.

\subsection{Metodología de la investigación}

El enfoque de esta investigación es cuantitativo. Este paradigma a guiado el tratamiento de los datos a través de la categorización y descripción de las propiedades, características y perfiles de las personas, grupos, comunidades, procesos y objetos que se han sometidos a análisis (Hernández et al. 2014). El diseño de esta investigación es "Ex Post Facto" porque este tipo de investigación trata de descubrir fenómenos que ocurren en forma natural, pero miden diversas variables para analizar su posible efecto (Bisquerra, 2012).

\subsection{Descripción de los participantes}

El estudio se llevó a cabo con una muestra de 163 estudiantes de Dowal School en los niveles de III ciclo de educación básica y educación media; pertenecientes a la zona del Distrito Central y zonas aledañas, en el departamento Francisco Morazán, Honduras. Dowal School es una institución educativa bilingüe de carácter privado con más de 30 años de experiencia en la formación y preparación de estudiantes, que van desde el nivel prebásico hasta educación media. 


\begin{tabular}{|l|l|l|l|}
\hline \multicolumn{4}{|c|}{ Tabla 1. Participantes de la Investigación } \\
\hline \multicolumn{1}{|c|}{ Grado } & Mujeres & Hombres & Total \\
\hline 7 th & 19 & 9 & 28 \\
\hline 8 th & 12 & 15 & 27 \\
\hline 9th & 16 & 21 & 37 \\
\hline 10th & 26 & 22 & 48 \\
\hline 11th & 16 & 7 & 23 \\
\hline Total & 89 & 74 & 163 \\
\hline
\end{tabular}

Entre los participantes del estudio el 55\% (89) son mujeres, frente al $45 \%$ (74) que son hombres. Respecto a su edad, la media se sitúa en 14.63 años con una desviación estándar de 1.456, encontrándose al $82 \%$ de los participantes en el intervalo de edad entre 13-16 años. En referencia a la variable grado académico: el 17.2\% son de séptimo grado; $16.6 \%$ son de octavo grado; $22.7 \%$ son de noveno grado; $29.4 \%$ de décimo grado; $14.1 \%$ de undécimo grado. Otra característica de la muestra es que el total de los estudiantes utilizan tecnologías de la información y comunicación para acceder a sus estudios virtuales, es decir, que el total de la población escolar utiliza computadoras, celulares o tabletas para recibir sus clases y realizar sus asignaciones, trabajos de clase y evaluaciones. Para concluir, hay que indicar que el tipo de muestreo que hemos utilizado ha sido el muestreo no probabilístico causal o accidental, que es aquel en el cual el investigador selecciona directa e intencionalmente la muestra, debido fundamentalmente a que tiene fácil acceso a la misma y es representativa de la población (Gil, Rodríguez \& García, 1995; Albert, 2006; Sabariego, 2004).

\subsection{Instrumentos de recogida de datos}

El instrumento para la recopilación de la información fue el cuestionario de actitudes hacia las matemáticas de Yáñez-Marquina \& Villardón-Gallego (2016). Según Yáñez-Marquina et al. (2016) el instrumento está constituido por 19 ítems, aglutinados en 3 dimensiones. Los estudiantes pueden indicar su agrado de acuerdo o desacuerdo con las afirmaciones propuestas por media de una escala Likert de 5 valores. Los factores asociados son: autoconcepto matemático de los estudiantes; utilidad percibida de las matemáticas; e interés hacia las matemáticas. La aplicación fue supervisada por los docentes de la clase y se pidió que a los estudiantes que respondieran de forma honesta y libre, ya que sus respuestas serian confidenciales. Es importante mencionar que el instrumento alcanzó un alfa de Cronbach de 0.804 lo que implica un nivel satisfactorio de confiabilidad del instrumento y la homogeneidad de los resultados obtenidos. 


\subsection{Procedimiento de administración y enfoque ético}

La administración del instrumento se realizó por parte de los autores durante el cuarto parcial (4to Trimestre) del año escolar 2020-2021 (2do Trimestre del 2do Semestre para los grados 10mo e 11mo). Tenía un carácter anónimo y fue completado por los sujetos participantes en presencia del profesorado. Con anterioridad a la toma de datos, se obtuvo tanto el consentimiento previo, libre e informado de los participantes, así como la autorización de las autoridades educativas.

\subsection{Análisis de datos}

Para satisfacer el objetivo del estudio se procedió al desarrollo de distintos análisis, empleando el paquete estadístico para las ciencias sociales SPSS v.25. Entre ellos, se aplicó un análisis de consistencia interna, un análisis descriptivo de los datos, un análisis de las correlaciones entre las categorías y pruebas paramétricas.

\section{Resultados}

Los datos obtenidos sobre el autoconcepto matemático muestran que los estudiantes se encuentran por encima de la media teórica (21) con un valor de 23.88, esto implica que se sienten capaces de disfrutar la clase de matemáticas y se han formado un autoconcepto positivo (Véase Tabla 2). Contrario a resultados previos como los de Ignacio et al. (2006) donde la mayoría de las mujeres muestran un deficiente autoconcepto como estudiantes de matemáticas y desconfían de su capacidad.

\begin{tabular}{|c|c|c|c|c|c|c|}
\hline \multirow[b]{2}{*}{ Criterios } & \multicolumn{5}{|c|}{ Respuestas \% } & \multirow[b]{2}{*}{$\begin{array}{l}\text { Puntuación } \\
\text { Media (SD) }\end{array}$} \\
\hline & $\begin{array}{l}\text { Fuerte- } \\
\text { mente en } \\
\text { desacu- } \\
\text { erdo }\end{array}$ & $\begin{array}{l}\text { En de- } \\
\text { sacuerdo }\end{array}$ & $\begin{array}{l}\text { Indife- } \\
\text { rente }\end{array}$ & $\begin{array}{l}\text { De acu- } \\
\text { erdo }\end{array}$ & $\begin{array}{l}\text { Fuerte- } \\
\text { mente de } \\
\text { acuerdo }\end{array}$ & \\
\hline $\begin{array}{l}\text { 1. Me siento más tonto que mis compañeros } \\
\text { de clase al resolver problemas y ejercicios de } \\
\text { matemáticas. }\end{array}$ & 14.1 & 13.5 & 28.2 & 22.1 & 22.1 & $3.25(1.324)$ \\
\hline $\begin{array}{l}\text { 2. A pesar de mis esfuerzos, no puedo en- } \\
\text { tender las matemáticas. }\end{array}$ & 6.1 & 13.5 & 22.7 & 28.2 & 29.4 & $3.61(1.214)$ \\
\hline 3. Tengo dificultades con las matemáticas. & 11.0 & 32.5 & 23.3 & 17.2 & 16.0 & $2.94(1.258)$ \\
\hline 4. No era un estudiante de matemáticas nato. & 22.7 & 19.6 & 19.6 & 22.7 & 15.3 & $2.88(1.394)$ \\
\hline $\begin{array}{l}\text { 5. Soy incapaz de resolver problemas } \\
\text { matemáticos }\end{array}$ & 3.1 & 6.7 & 24.5 & 28.2 & 37.4 & $3.90(1.078)$ \\
\hline $\begin{array}{l}\text { 6. Haga lo que haga, obtengo bajas califica- } \\
\text { ciones en matemáticas. }\end{array}$ & 9.2 & 7.4 & 19.6 & 27.6 & 36.2 & $3.74(1.275)$ \\
\hline $\begin{array}{l}\text { 7. Siempre será difícil para mí aprender } \\
\text { matemáticas. }\end{array}$ & 10.4 & 8.6 & 27.6 & 22.1 & 31.3 & $3.55(1.296)$ \\
\hline \multicolumn{7}{|l|}{ Puntuación Total ${ }^{a}$} \\
\hline
\end{tabular}


Tabla 2. Autoconcepto matemático de los estudiantes

\begin{tabular}{|c|c|c|c|c|c|c|}
\hline \multirow[b]{2}{*}{ Criterios } & \multicolumn{5}{|c|}{ Respuestas \% } & \multirow[b]{2}{*}{$\begin{array}{l}\text { Puntuación } \\
\text { Media (SD) }\end{array}$} \\
\hline & $\begin{array}{l}\text { Fuerte- } \\
\text { mente en } \\
\text { desacu- } \\
\text { erdo }\end{array}$ & $\begin{array}{l}\text { En de- } \\
\text { sacuerdo }\end{array}$ & $\begin{array}{l}\text { Indife- } \\
\text { rente }\end{array}$ & $\begin{array}{c}\text { De acu- } \\
\text { erdo }\end{array}$ & $\begin{array}{l}\text { Fuerte- } \\
\text { mente de } \\
\text { acuerdo }\end{array}$ & \\
\hline Mediana (rango) & \multicolumn{6}{|c|}{$24.0000(11-32)$} \\
\hline Media (SD) & \multicolumn{6}{|c|}{$23.8834(5.186)$} \\
\hline Varianza & \multicolumn{6}{|l|}{26.894} \\
\hline Puntuación total \% & \multicolumn{6}{|l|}{$68.23 \%$} \\
\hline
\end{tabular}

a Máxima puntuación $=35$

En cuanto a la utilidad percibida de las matemáticas, los resultados muestran que los estudiantes consideran útil el aprendizaje de las matemáticas para entender otras asignaturas y como elementos necesarios para la vida profesional (Véase Tabla 3), coincidiendo con Caballero-Carrasco, Blanco-Nieto y Guerrero-Barona (2008) quienes en su estudio con estudiantes para maestro concluyen que consideran las matemáticas como útiles y necesarias tanto para desenvolverse adecuadamente en la sociedad como para asimilar y dominar otras asignaturas que guardan relación con dicha disciplina.

Tabla 3. Utilidad percibida de las matemáticas

\begin{tabular}{|c|c|c|c|c|c|c|}
\hline \multirow[b]{2}{*}{ Criterios } & \multicolumn{5}{|c|}{ Respuestas \% } & \multirow[b]{2}{*}{$\begin{array}{l}\text { Puntuación } \\
\text { Media (SD) }\end{array}$} \\
\hline & $\begin{array}{l}\text { Fuertemente } \\
\text { en desacuerdo }\end{array}$ & $\begin{array}{c}\text { En } \\
\text { desacu- } \\
\text { erdo }\end{array}$ & $\begin{array}{l}\text { Indife- } \\
\text { rente }\end{array}$ & $\begin{array}{l}\text { De acu- } \\
\text { erdo }\end{array}$ & $\begin{array}{l}\text { Fuerte- } \\
\text { mente de } \\
\text { acuerdo }\end{array}$ & \\
\hline 8. Las matemáticas son muy útiles. & 0.6 & 6.7 & 14.7 & 30.7 & 47.2 & $4.17(0.960)$ \\
\hline $\begin{array}{l}\text { 9. Todo el mundo necesita aprender } \\
\text { matemáticas. }\end{array}$ & 0.6 & 3.7 & 12.3 & 25.2 & 58.3 & $4.37(0.882)$ \\
\hline $\begin{array}{l}\text { 10. Las matemáticas son necesarias } \\
\text { para la vida. }\end{array}$ & 0.6 & 4.9 & 14.7 & 31.3 & 48.5 & $4.22(0.916)$ \\
\hline $\begin{array}{l}\text { 11. Las matemáticas son importantes } \\
\text { para el desarrollo de la sociedad. }\end{array}$ & 1.2 & 5.5 & 16.6 & 31.3 & 45.4 & $4.14(0.968)$ \\
\hline $\begin{array}{l}\text { 12. Aprender matemáticas es impor- } \\
\text { tante para mi futuro trabajo. }\end{array}$ & 3.7 & 12.9 & 25.2 & 20.2 & 38.0 & $3.76(1.196)$ \\
\hline \multicolumn{7}{|l|}{ Puntuación Total a } \\
\hline Mediana (rango) & \multicolumn{6}{|l|}{$22.0000(10-25)$} \\
\hline Media (SD) & \multicolumn{6}{|c|}{$20.6626(3.75026)$} \\
\hline Varianza & \multicolumn{6}{|l|}{14.064} \\
\hline Puntuación total \% & \multicolumn{6}{|l|}{$59.036 \%$} \\
\hline
\end{tabular}

a Máxima puntuación $=25$

Con respecto al interés hacia las matemáticas se observa que los estudiantes sienten poco interés a pesar de reconocer su utilidad y que se consideran matemáticamente capaces (Véase Tabla 4). 


\begin{tabular}{|c|c|c|c|c|c|c|}
\hline \multirow[b]{2}{*}{ Criterios } & \multicolumn{5}{|c|}{ Respuestas \% } & \multirow[b]{2}{*}{$\begin{array}{l}\text { Puntuación } \\
\text { Media (SD) }\end{array}$} \\
\hline & $\begin{array}{c}\text { Fuerte- } \\
\text { mente en } \\
\text { desacuerdo }\end{array}$ & $\begin{array}{c}\text { En } \\
\text { desacu- } \\
\text { erdo }\end{array}$ & $\begin{array}{l}\text { Indife- } \\
\text { rente }\end{array}$ & $\begin{array}{l}\text { De acu- } \\
\text { erdo }\end{array}$ & $\begin{array}{l}\text { Fuerte- } \\
\text { mente } \\
\text { de acu- } \\
\text { erdo }\end{array}$ & \\
\hline 13. Me gusta estudiar matemáticas & 23.3 & 20.2 & 37.4 & 14.7 & 4.3 & $2.56(1.128)$ \\
\hline 14. Me gustan las matemáticas & 19.6 & 21.5 & 31.3 & 17.8 & 9.8 & $2.77(1.235)$ \\
\hline $\begin{array}{l}\text { 15. El tiempo pasa volando cuando es- } \\
\text { tudio matemáticas }\end{array}$ & 25.8 & 25.2 & 22.7 & 16.6 & 9.8 & $2.60(1.299)$ \\
\hline 16. Estudiar matemáticas es divertido. & 30.1 & 30.1 & 25.8 & 11.0 & 3.1 & $2.27(1.100)$ \\
\hline $\begin{array}{l}\text { 17. El tiempo pasa volando cuando re- } \\
\text { suelvo problemas y ejercicios matemáti- } \\
\text { cos. }\end{array}$ & 21.5 & 26.4 & 24.5 & 16.0 & 11.7 & $2.70(1.292)$ \\
\hline 18. Las matemáticas son entretenidas. & 22.7 & 22.7 & 30.7 & 17.2 & 6.7 & $2.63(1.202)$ \\
\hline 19. Las matemáticas son una molestia. & 8.6 & 27.0 & 35.6 & 15.3 & 13.5 & $2.98(1.147)$ \\
\hline \multicolumn{7}{|l|}{ Puntuación Total a $^{a}$} \\
\hline Mediana (rango) & \multicolumn{6}{|c|}{$18.0000(7-35)$} \\
\hline Media (SD) & \multicolumn{6}{|c|}{$18.5031(5.3036)$} \\
\hline Varianza & \multicolumn{6}{|l|}{28.128} \\
\hline Puntuación total \% & \multicolumn{6}{|l|}{$52.866 \%$} \\
\hline
\end{tabular}

a Máxima puntuación $=35$

En relación con el análisis de correlaciones muestra una correlación positiva entre estos tres componentes (Tabla 5), es decir el autoconcepto positivo tiene relación directa con el aumento en el interés y la utilidad percibida de las matemáticas por los estudiantes.

\begin{tabular}{|l|c|c|c|}
\hline \multicolumn{4}{|c|}{ Tabla 5: Análisis de correlaciones entre los factores (r-Pearson) } \\
\hline \multicolumn{1}{|c|}{ Factores } & Categoría 1 & Categoría 2 & Categoría 3 \\
\hline $\begin{array}{l}\text { 1. Autoconcepto matemático de los estudi- } \\
\text { antes }\end{array}$ & 1 & 0.187 & 0.448 \\
\hline 2. Utilidad percibida de las matemáticas & 0.187 & 1 & 0.233 \\
\hline 3. Interés hacia las matemáticas & 0.448 & 0.233 & 1 \\
\hline
\end{tabular}

En relación con el análisis paramétrico se encontró que la variable género $(\mathrm{p}=0.072)$, edad ( $\mathrm{p}=0.184)$, grado $(\mathrm{p}=0.069)$ o sección $(\mathrm{p}=0.104)$ no son determinantes porque no se encontraron diferencias significativas, es decir, el valor de la significancia es mayor que 0.05. Tampoco, se encontró diferencias significativas entre las variables género $(p=0.232)$, edad $(p=0.289)$, grado $(p=0.571)$ o sección $(p=0.179)$ con respecto a la utilidad percibida de las matemáticas. Así mismo, se muestra que no existen diferencias significativas en cuanto al género $(p=0.652)$, edad $(p=0.063)$, grado $(p=0.408)$ o sección $(\mathrm{p}=0.744)$ en referencia al interés hacia las matemáticas. Incluso de forma global en las actitudes no se encontró diferencias significativas con respecto a género $(\mathrm{p}=0.381)$, edad $(\mathrm{p}=0.123)$, grado $(\mathrm{p}=0.906)$ o sección $(\mathrm{p}=0.308)$. Por lo que se puede 


\section{INVESTIGACIÓN EDUCATIVA}

decir que ninguna de estas variables es categórica en las actitudes de los estudiantes sean estas positivas o negativas.

\begin{tabular}{|c|c|c|c|c|}
\hline \multirow[t]{2}{*}{ Factores } & Género & Edad & $\begin{array}{c}\text { Grado Aca- } \\
\text { démico }\end{array}$ & Sección \\
\hline & $p$ & $p$ & $p$ & $p$ \\
\hline $\begin{array}{l}\text { Autoconcepto matemático de } \\
\text { los estudiantes }\end{array}$ & 0.072 & 0.184 & 0.069 & 0.104 \\
\hline $\begin{array}{l}\text { Utilidad percibida de las } \\
\text { matemáticas }\end{array}$ & 0.232 & 0.289 & 0.571 & 0.179 \\
\hline Interés hacia las matemáticas & 0.652 & 0.063 & 0.408 & 0.744 \\
\hline $\begin{array}{l}\text { Actitudes globales hacia las } \\
\text { matemáticas }\end{array}$ & 0.384 & 0.123 & 0.906 & 0.306 \\
\hline
\end{tabular}

\section{Discusión y conclusiones}

Las actitudes globales hacia las matemáticas muestran una media de 63.05\%, atendiendo los resultados de las actitudes globales se puede concluir que las metodologías utilizadas en el proceso de aprendizaje han sido efectivas para que los estudiantes aprendan matemáticas y resolver prácticas matemáticas.

Los estudiantes tienen un autoconcepto matemático positivo con una media de 23.88, el cual supera a la media teórica (21) en este aspecto, en segundo lugar, los estudiantes perciben que las matemáticas son útiles representado por una media 20.66 en este aspecto (cuya media teórica es 15), el tercer aspecto actitudinal es el que contrasta con los datos de los primeros dos, ya que los estudiantes muestran poco interés hacia las matemáticas, evidenciado por una media de 18.50 que está por debajo de la media teórica (21), esto implica que aun cuando los estudiantes se sienten capacitados para aprender matemáticas y la consideran útil e importante, no todos muestran interés hacia la misma.

Aunque el resultado de este último aspecto (interés) parezca desesperanzador, los datos arrojan correlaciones positivas entre el autoconcepto, la utilidad percibida y el interés, razón por la cual, aunque 18.50 está por debajo de la media, es estadísticamente similar a las medias de los otros dos aspectos, en adición a esto el análisis global de todos los ítems arroja una media de 63.04 y una desviación estándar de 10.50, valor que está por encima de la media teórica (57) implicando que los estudiantes participantes en el estudio tienen una actitud positiva con respecto al estudio de las matemáticas.

Por otro lado, al analizar las variables género, edad, grado académico y sección se ha notado que estas no implican una diferencia a la hora de determinar las actitudes, esto significa, que las matemáticas son consideradas importantes por los alumnos sin importar su grado, lo que es coherente con los resultados obtenidos por Roca y 
Díez-Palomar (2011) quienes mencionan no haber encontrado evidencia de la existencia de un patrón de relación entre edad y actitud frente a las matemáticas en grupos de edades más dispersos. En este mismo sentido, cabe mencionar que, al no encontrarse diferencia significativa entre los grupos formados por estas variables demográficas, se concluye que la población estudiantil es bastante homogénea, un caso que llama la atención ya que, es normal suponer algún tipo de heterogeneidad entre diferentes conjuntos de seres humanos, esta homogeneidad es entendible debido a los procesos de admisión del centro educativo en cuestión y que, al parecer, los docentes tienen estilos de enseñanza y actitudes similares hacia las matemáticas.

En definitiva, es necesario seguir avanzando en la utilización de ambientes de aprendizaje para la enseñanza de las matemáticas, porque constituyen un recurso

útil para favorecer procesos de aprendizaje de las matemáticas en contexto multiculturales y plurilingües, por consiguiente, es necesario avanzar en la formación del profesorado, la construcción de ambientes de aprendizaje, el reconocimiento de la diversidad y la resiliencia afectiva e intercultural en el aprendizaje de las matemáticas (Flores-López, 2021).

\section{Lista de referencia}

Albert, M. (2006). La investigación educativa. Claves teóricas. Madrid: MCGraw-Hill.

Bisquerra, R., \& Alzina, R. B. (2012). Metodología de la investigación educativa. Editorial La Muralla.

Caballero-Carrasco, A., Blanco-Nieto, L., Guerrero-Barona, E. (2008). El dominio afectivo en futuros maestros de matemáticas en la Universidad de Extremadura. Paradigma, 29(2), 157-171. https://bit.ly/3d3Lfsv

Flores-López, W. O. (2021). Ambiente de aprendizaje para la enseñanza de las matemáticas ante el COVID-19. Ciencia e Interculturalidad, 28(01), 9 - 22. https:// doi.org/https://doi.org/10.5377/10.5377/rci.v28io1.11455

Flores-López, W.O \& Auzmendi, E. (2018). Actitudes hacia las matemáticas en la enseñanza universitaria y su relación con las variables género y etnia. Profesorado. Revista de Currículum y Formación de Profesorado, 22(3), 231-251. https://doi. org/10.30827/profesorado.v22i3.8000

García-Soto, Y. L., Flores-López, W. O., \& Olivar Molina, S. A. (2018). Competencias emocionales en la formación del profesorado de educación secundaria y su relación con las actitudes hacia las matemáticas. Revista Electrónica de Conocimientos, Saberes y Prácticas, 1(2). https://doi.org/10.30698/recsp.v1i2.9 


\section{INVESTIGACIÓN EDUCATIVA}

Gil, J., Rodríguez, G., y García, E. (1995). Estadística básica aplicada a las Ciencias de la Educación. Sevilla: Kronos.

Hernández, R., Baptista Lucio, P., \& Fernández Collado, C. (2014). Metodología de la Investigación. Editorial McGraw Hill.

Ignacio, N. G. (2006). The Affective Domain in Mathematics Learning. International Electronic Journal of Mathematics Education, 16-32.

Jiménez-Bonilla, E., \& Flores-López, W. O. (2017). Actitudes hacia las matemáticas: un estudio en una escuela rural de la Costa Caribe Sur de Nicaragua. Revista Universitaria del Caribe, 18(1), 7-16. https://doi.org/10.5377/ruc.v18i1.4794

OCDE. (2018). PISA para el Desarrollo: Resultados en Foco. https://bit.ly/3d5bdeV

PISA. (2018). Dirección General de Currículo y Evaluación: La educación en Honduras: Hallazgos en Honduras por su participación en PISA para el Desarrollo. Secretaría de Educación de Honduras. https://bit.ly/3zRcOyY

Roca, A. E., \& Díez-Palomar, J. (2011). Las actitudes hacia las Matemáticas. Análisis descriptivo de un estudio de caso exploratorio centrado en la Educación Matemática de familiares. Revista de investigación en educación, 9(2), 116-132.

Rocha-Feregrino, G., Fuchs-Gómez, O. L., \& Juárez-López, J. A. (2021). Importancia del estudio de las actitudes para el aprendizaje de las matemáticas. RD-ICUAP, 7(19), 148-157. https://bit.ly/3vTRky6

Sabariego, M. (2004). El proceso de investigación. En R. Bisquerra, Metodología de la investigación educativa (127-163). Madrid: La Muralla.

Solís, M. (2017). El afecto y el aprendizaje. Análisis de una discusión colegiada en el seminario repensar las matemáticas del IPN. Experiencias Educativas Innovadoras, 96-104. https://bit.ly/2SWSlij

TIMSS \& PIRLS International Study Center. (2011). TIMSS 2011 International Results in Mathematics. IEA International Association for the Evaluation of Educational Achievement. https://bit.ly/3j1cAiN

Yáñez-Marquina, L., \& Villardón-Gallego, L. (2016). Attitudes towards mathematics at secondary level: Development and structural validation of the Scale for Assessing Attitudes towards Mathematics in Secondary Education (SATMAS). Electronic Journal of Research in Educational Psychology, 14(3), 557-581. https:// doi.org/10.14204/ejrep.40.15163 